\title{
Triphenylsilyl- and Trityl-Substituted Carbazole-Based Host Materials for Blue Electrophosphorescence
}

\author{
Ming-Han Tsai, ${ }^{\dagger}$ Tung-Huei Ke, ${ }^{\dagger}$ Hao-Wu Lin, ${ }^{\dagger}$ Chung-Chih Wu, ${ }^{*}{ }^{\dagger}$ Shih-Feng Chiu, ${ }^{\ddagger}$ \\ Fu-Chuan Fang, ${ }^{\mp}$ Yuan-Li Liao, ${ }^{\ddagger}$ Ken-Tsung Wong, ${ }^{*},{ }^{\dagger}$ Yu-Hung Chen, ${ }^{\dagger}$ and Chih-I Wu ${ }^{\dagger}$
}

Department of Electrical Engineering, Graduate Institute of Electro-optical Engineering and Graduate Institute of Electronics Engineering, and Department of Chemistry, National Taiwan University, Taipei, Taiwan 106

\begin{abstract}
Carbazole-based materials adopting the nonconjugated substitution of triphenylsilyl $\left(-\mathrm{SiPh}_{3}\right)$ and trityl $\left(-\mathrm{CPh}_{3}\right) \operatorname{side}$ groups are studied as high-triplet-energy, morphologically, and electrochemically stable host materials with tunable carrier-transport properties for organic blue electrophosphorescence. The developed host materials 9-(4-tert-butylphenyl)-3,6-bis(triphenylsilyl)-9H-carbazole (CzSi), 9-(4-tert-butylphenyl)-3,6-ditrityl-9H-carbazole (CzC), and 9-(4-tert-butylphenyl)-3-(triphenylsilyl)-6-trityl-9H-carbazole (CzCSi) all show high triplet energies of $2.97-3.02 \mathrm{eV}$, along with high glass transition temperatures of $131-163{ }^{\circ} \mathrm{C}$ and superior electrochemical stability. Nevertheless, the carrier-transport properties show rather significant dependence on different substitutions. Although three different host materials give similar peak electroluminescence efficiencies at low driving currents, the CzSi host, which has more suitable carrier-transport properties, renders broadened distributions of the triplet excitons in phosphorescent devices, reducing the quenching associated with triplet-triplet annihilation and giving larger resistance against efficiency roll-off at higher brightnesses.
\end{abstract}

KEYWORDS: blue electrophosphorescence $\bullet$ host $\bullet$ carbazole $\bullet$ triphenylsilyl substitution $\bullet$ trityl substitution $\bullet$ triplet energy $\bullet$ charge transport

\section{INTRODUCTION}

he development of efficient phosphorescent organic
light-emitting devices (OLEDs) in recent years renders
possible harvesting of both electrogenerated singlet and triplet excitons for realizing nearly $100 \%$ internal quantum efficiencies of electroluminescence (EL) (1-8). While green and red phosphorescent OLEDs have been demonstrated to achieve nearly $100 \%$ internal quantum efficiency (2), research efforts to improve the performances of blue phosphorescent OLEDs are still highly desired. In pursuing highly efficient phosphorescent OLEDs, it is essential that the triplet energy of the host be larger than that of the triplet emitter to prevent reverse energy transfer from the guest back to the host and to effectively confine triplet excitons on guest molecules. Such a requirement becomes particularly challenging when blue electrophosphorescence is of interest (3-8), in which the conjugation lengths of host molecules must be extremely confined to achieve a triplet energy level larger than photon energies of blue light (i.e., $\geq 2.7 \mathrm{eV}$ ). As such, host materials reported for blue electrophosphorescence have thus far been rare (4-8). Among them, tetraphenylsilane derivatives (such as UGH materials) and carbazole-based materials are two common classes of

*E-mail: chungwu@cc.ee.ntu.edu.tw(C.-C.W), kenwong@ntu.edu.tw (K.-T.W.).

Received for review October 15, 2008 and accepted January 6, 2009

${ }^{\dagger}$ Department of Electrical Engineering, Graduate Institute of Electro-optical Engineering and Graduate Institute of Electronics Engineering.

* Department of Chemistry

DOI: $10.1021 / \mathrm{am} 800124 \mathrm{q}$

๑ 2009 American Chemical Society large-triplet-energy host materials for blue electrophosphorescence. Although tetraphenylsilane derivatives possess particularly large triplet energies of well over $3.0 \mathrm{eV}$, they, however, usually suffer poor conductivity/carrier-transport properties and poor morphological stabilities (i.e., low glass transition temperatures) (8), which are detrimental to either the device efficiency/operation voltage or long-term stability. On the other hand, materials based on the carbazole moiety are more electrically active systems (4-7). Carbazole moieties are characterized by their relatively small exchange energies, which are associated with small orbital overlaps in their $n-\pi^{*}$ transitions (9-11). The exchange energies of carbazole-based compounds can be as small as about 0.3-0.5 eV for judiciously designed molecular structures (9-11), thus rendering high triplet energies. Small exchange energies make a significant contribution to the reduction of the energy loss in the excitation of dopants via conductive hosts, which would improve the power efficiency of the device.

To achieve a large triplet energy, successive $\pi$ conjugation beyond one monomer (e.g., a single phenyl ring or carbazole unit) is generally undesirable (9-11). This, however, conflicts with film morphological stability considerations because materials with molecular sizes as small as monomers usually suffer from the tendency of crystallization and thermal instability. For molecules to form morphologically stable and uniform amorphous films with typical processing techniques, the molecule size must be extended beyond one carbazole monomer to afford bulky and steric molecular configurations, yet without reduction of the triplet energy of the molecule. Previously, we had reported that such 
Scheme 1. Synthesis of $\mathrm{CzSi}, \mathrm{CzC}$, and $\mathrm{CzCSi}$

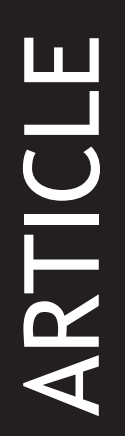

challenges can be conquered by introducing triphenylsilyl onto the $\mathrm{C} 3$ and $\mathrm{C} 6$ sites of carbazole as sterically hindered side groups, forming a molecule of 9-(4-tert-butylphenyl)-3,6bis(triphenylsilyl)-9H-carbazole (CzSi) with enhanced morphological stability (12). The tetrahedral $\mathrm{Si}$ atoms serve as effective spacers, blocking the $\pi$ conjugation of the carbazole core from extending to the peripheral substitution; thus, the large triplet energy of carbazole can be retained. Rather efficient blue phosphorescent OLEDs had been demonstrated using such a host material.

In our previous report, properties of $\mathrm{CzSi}$ were not thoroughly characterized, and thus it is not well understood why $\mathrm{CzSi}$ is successful in giving highly efficient blue phosphorescent devices, particularly when considering that its triplet energy is similar to those of other reported carbazolebased wide-gap host materials. To understand the critical and fundamental issues (in addition to triplet energies, glass transition temperatures, etc.) in the design of effective host materials for blue organic electrophosphorecence, in this paper we systematically vary the nonconjugated substitutions on C3 and C6 of carbazole, such as triphenylsilyl, trityl, or their combinations, to tune the properties of the host materials while still maintaining their large triplet energies. These carbazole-based host materials include $\mathrm{CzSi}$, 9-(4-tertbutylphenyl)-3,6-ditrityl-9H-carbazole (CzC), and 9-(4-tertbutylphenyl)-3-(triphenylsilyl)-6-trityl-9H-carbazole (CzCSi).

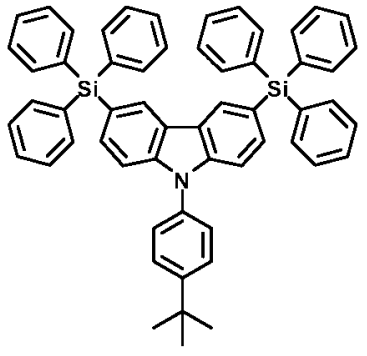

CzSi
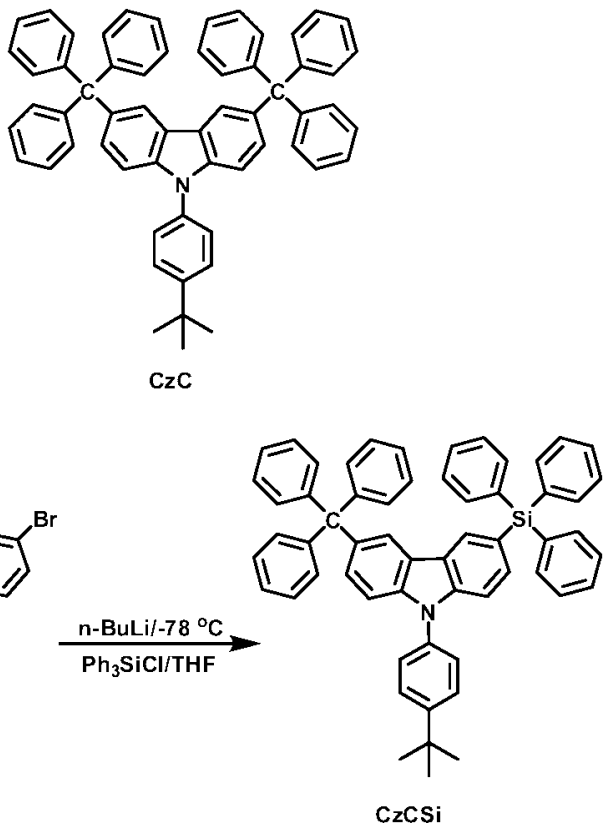

By thoroughly characterizing various properties (e.g., chemical, electrochemical, thermal, photophysical, charge-transport, and electroluminescent properties, energy levels, etc.) of these host materials, we seek to understand the critical and fundamental issues that need to be considered in the selection and design of effective host materials for blue organic electrophosphorecence, in addition to the more commonly recognized requirements in triplet energies, glass transition temperatures, etc.

\section{RESULTS AND DISCUSSION}

2.1. Synthesis. The synthetic pathways of the target compounds $\mathrm{CzSi}, \mathrm{CzC}$, and $\mathrm{CzCSi}$ are depicted in Scheme 1. The 3,6-dibromo derivatives of carbazole were treated with $n$-BuLi at $-78{ }^{\circ} \mathrm{C}$ to give dilithiated intermediates, which were subsequently quenched with chlorotriphenylsilane to give the desired $\mathrm{CzSi}$ in $78 \%$ yield after column chromatography. 9-(4-tert-Butylphenyl)carbazole and triphenylmethanol were dissolved in $\mathrm{CH}_{2} \mathrm{Cl}_{2}$, and Eaton's reagent (7.7 wt \% $\mathrm{P}_{2} \mathrm{O}_{5}$ in $\mathrm{CH}_{3} \mathrm{SO}_{3} \mathrm{H}$ ) was added dropwise at $25^{\circ} \mathrm{C}$ under a nitrogen atmosphere to give the desired $\mathrm{CzC}$ in $84 \%$ yield. Compound $\mathbf{1}$ prepared similarly to the synthesis of $\mathrm{CzC}$ was dissolved in $\mathrm{CH}_{2} \mathrm{Cl}_{2}$, and a solution of $\mathrm{N}$-bromosuccinimide in dimethylformamide was added slowly at room temperature to give compound $\mathbf{2}$. Compound $\mathbf{2}$ was treated 

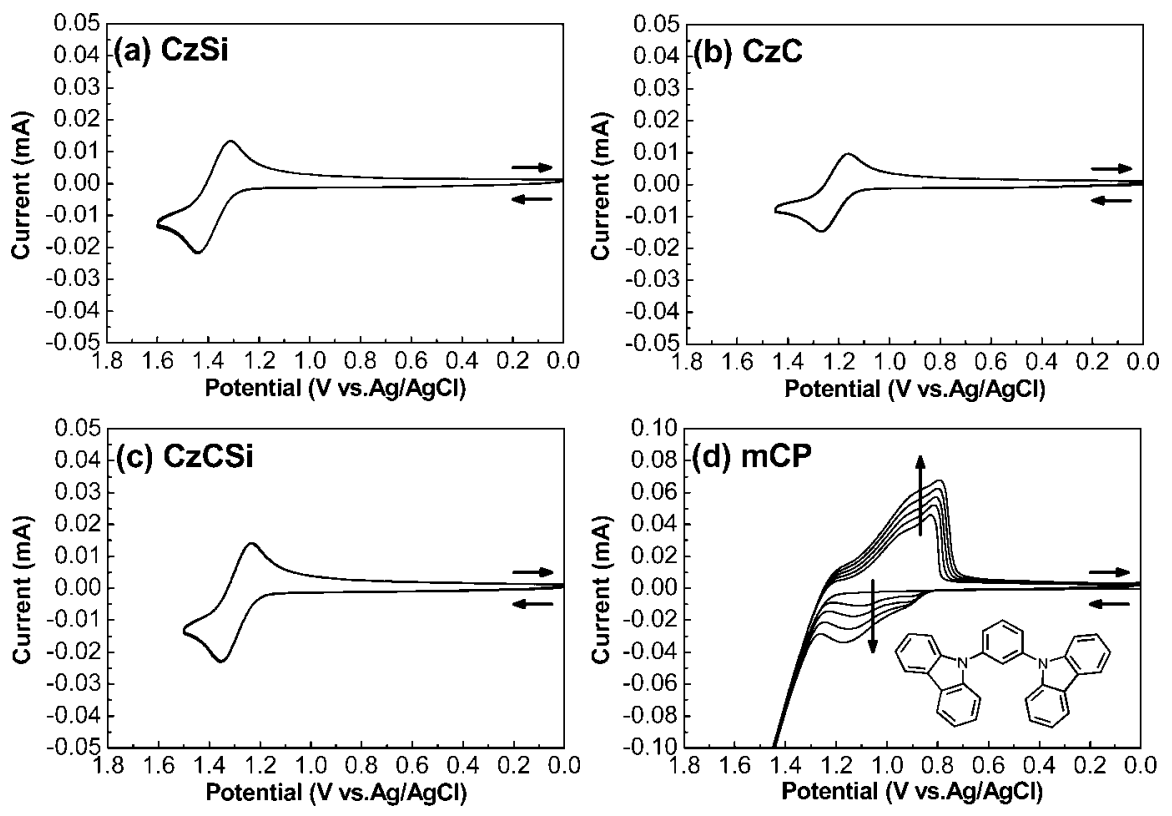

FIGURE 1. Repeated cyclic voltammograms of (a) CzSi (five cycles), (b) CzC (five cycles), (c) CzCSi (five cycles), and (d) mCP (five cycles). The inset of part $d$ is the molecular structure of $\mathrm{mCP}$.

with $n$-BuLi at $-78{ }^{\circ} \mathrm{C}$ and subsequently quenched with chlorotriphenylsilane to give $\mathrm{CzCSi}$ in $60 \%$ yield.

2.2. Cyclic Voltammetry (CV). The electrochemical properties of the compounds $\mathrm{CzSi}, \mathrm{CzC}$, and $\mathrm{CzCSi}$ were investigated by $\mathrm{CV}$, as shown in Figure $1 \mathrm{a}-\mathrm{c}$. In $\mathrm{CH}_{2} \mathrm{Cl}_{2}$, using $0.1 \mathrm{M} n-\mathrm{Bu}_{4} \mathrm{NPF}_{6}$ as the supporting electrolyte, a glassy carbon electrode as the working electrode, and $\mathrm{Ag} / \mathrm{AgCl}$ as the reference electrode with the scan rate set to $100 \mathrm{mV} / \mathrm{s}$, reversible oxidation was observed. Therefore, it is reasonable to expect that the present host compounds be stable upon accepting holes in OLEDs. In contrast, when the electrochemically active sites (C3 and C6 of carbazoles) are left unblocked, the oxidation process of the commonly used blue phosphorescent OLED host mCP ( $N, N^{\prime}$-dicarbazolyl-3,5benzene; inset of Figure $1 \mathrm{~d}$ ) is not reversible (4), with the oxidation potential gradually shifting to lower potentials and the current increasing during repeated CV scans, as shown in Figure $1 \mathrm{~d}$. As revealed in the literature, such characteristics are signatures of electrochemical polymerization of carbazoles through the active C3 and C6 sites $(10,13,14)$. These electrochemical results indicate that the introduction of triphenylsilyl and trityl substitutions brings enhanced electrochemical stability because they block the electrochemically active sites (C3 and C6) of carbazole. This is an important factor in the application of carbazole compounds in OLEDs when the compounds transport positive charge carriers in the devices.

Figure 2 depicts the phosphorescence spectra of pristine films deposited by thermal evaporation compared with films on the indium-tin oxide (ITO) working electrode during repeating CV scans. It is evident that, as a result of electrochemical polymerization, new species with extended conjugation lengths are formed in CV-deposited films of $\mathrm{mCP}$. The new chromophores exhibit reduced triplet energies in the region from about 500-600 nm (Figure 2b). In contrast, CzSi with the electrochemically active sites of carbazole

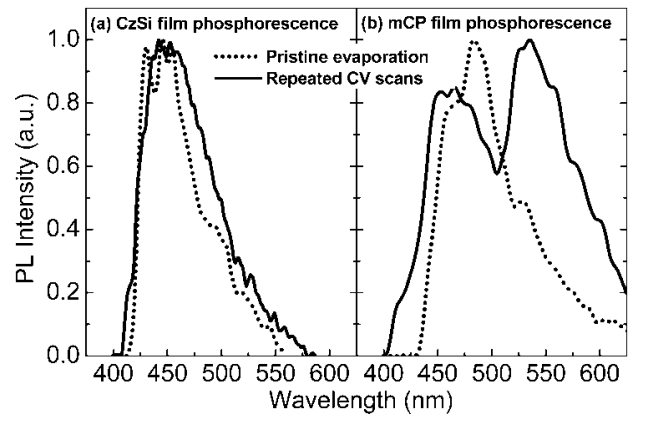

FIGURE 2. Phosphorescence of pristine films deposited by thermal evaporation compared with the films on the ITO working electrode in repeated CV scans of (a) $\mathrm{CzSi}$ and (b) $\mathrm{mCP}$.

protected does not undergo electrochemical polymerization but merely adsorbs on the ITO electrode. The phosphorescence spectrum of $\mathrm{CzSi}$ in pristine evaporated film and that adsorbed on the ITO working electrode are nearly identical, as shown in Figure 2a. Therefore, it is capable of suppressing the formation of reduced triplet energy species. Such results provide further evidence of the superior electrochemical stability of the developed host materials.

2.3. Thermal Properties. As mentioned above, the introduction of triphenylsilyl and trityl groups to C3 and C6 of carbazole renders the molecule rather steric, rigid, and bulky. Such a molecular configuration is strongly beneficial to the thermal stability, as indicated by the high decomposition temperature ( $T_{\mathrm{d}}$, corresponding to $5 \%$ weight loss in the thermogravimetric analysis, TGA) of 392,393 , and $388^{\circ} \mathrm{C}$ for $\mathrm{CzSi}, \mathrm{CzC}$, and $\mathrm{CzCSi}$, respectively. The glass transition temperatures $\left(T_{\mathrm{g}}\right)$ determined by differential scanning calorimetry (DSC) are 131,163 , and $146^{\circ} \mathrm{C}$ for $\mathrm{CzSi}, \mathrm{CzC}$, and CzCSi, respectively. Such $T_{\mathrm{g}}$ 's are rather high and are more than 2 times higher than that of $\mathrm{mCP}\left(T_{\mathrm{g}}=55^{\circ} \mathrm{C}\right)(7)$. The $T_{\mathrm{g}}$ 'S are in the order of $\mathrm{CzC}>\mathrm{CzCSi}>\mathrm{CzSi}$, where the trityl substitutions provide higher morphological stability (higher $T_{\mathrm{g}}$ ) than the triphenylsilyl substitutions. This might be at- 

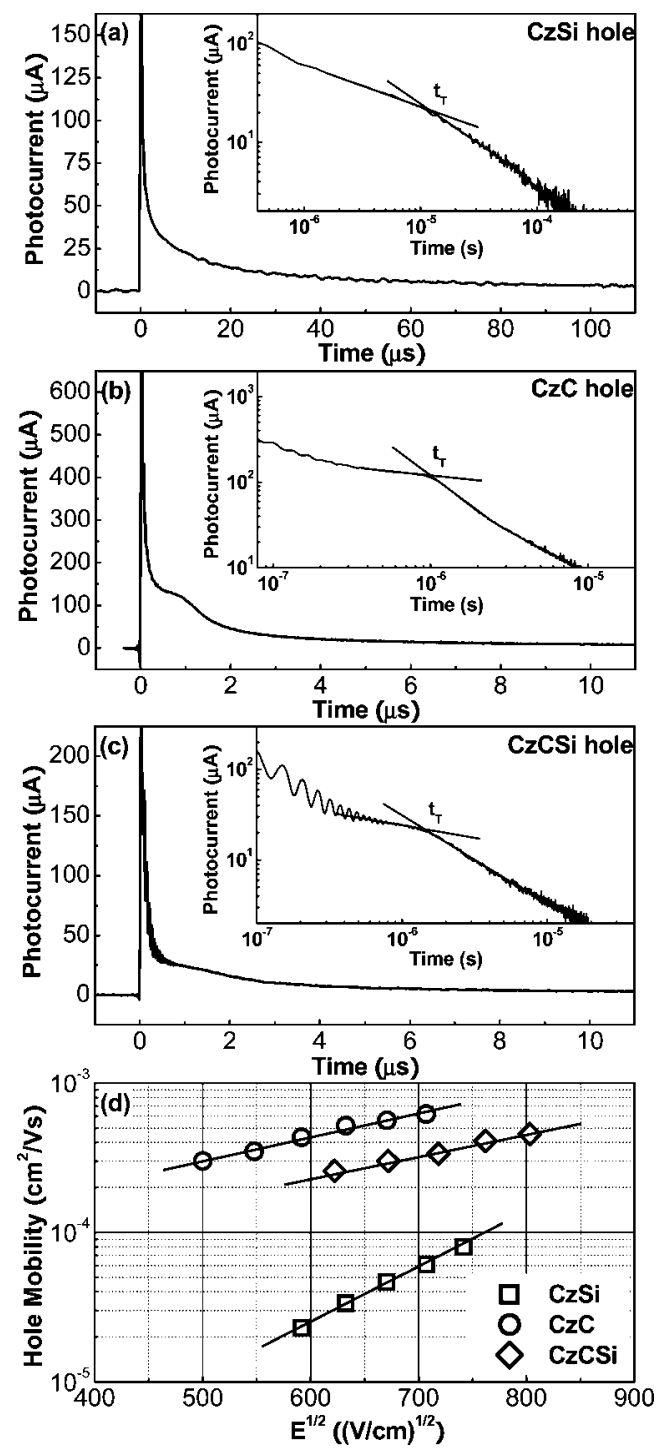

FIGURE 4. TOF transients of (a) $\mathrm{CzSi}$, (b) $\mathrm{CzC}$, and (c) $\mathrm{CzCSi}$. Insets: double-logarithmic plots. (d) Hole mobilities of the host materials vs square roots of the electric field.

of the phosphorescent devices, which will be discussed in the following section.

2.6. Device Results. The developed host compounds $\mathrm{CzSi}, \mathrm{CzC}$, and $\mathrm{CzCSi}$ have been subjected to electrophosphorescence studies. The devices were fabricated on glass substrates with the typical structure of multiple organic layers sandwiched between the bottom ITO anode and the top metal cathode (Al). The device structure used was ITO/ PEDT-PSS $(\sim 300 \AA) / D P A S(175 \AA) / T C T A(25 \AA) /$ host doped with 8 wt \% FIrpic $(250 \AA) / T A Z(500 \AA) / L i F(5 \AA) / A l(1000$ $\AA$ ), where the conducting polymer poly(ethylenedioxythiophene)/poly(styrene sulfonate) (PEDT-PSS) was used as the hole-injection layer (16), 2,2'-bis( $N, N$-diphenylamine)9,9'-spirobifluorene (DPAS) and 4,4',4"'-tri- $N$-carbazolyltriphenylamine (TCTA) as the hole-transport layers (12), host compounds with a nearly optimized concentration (8 wt \%) of the blue phosphorescent dopant iridium(III) bis[4,6difluorophenyl]pyridinato- $N, C^{2}$ ]picolinate (FIrpic) as the emitting layer $(3-5,7), 3$-(4-biphenylyl)-4-phenyl-5-(4-tert-butylphenyl)-1,2,4-triazole (TAZ) as the electron-transport layer (a)
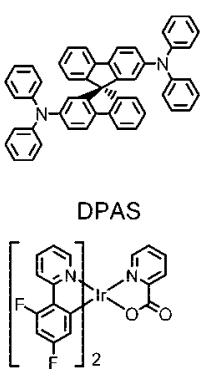

Flrpic
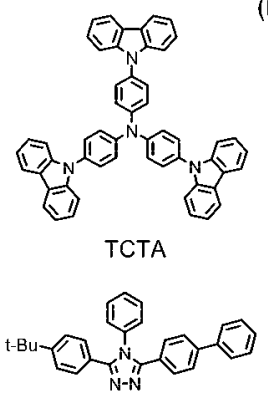

TAZ (b)

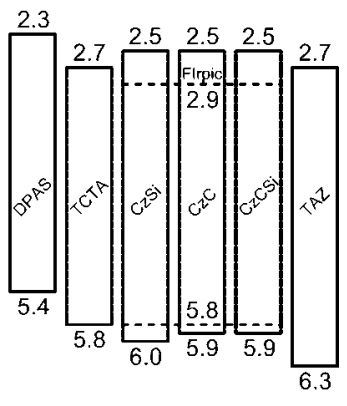

FIGURE 5. (a) Chemical structures of DPAS, TCTA, FIrpic, and TAZ. (b) Energy levels of related compounds in thin films.

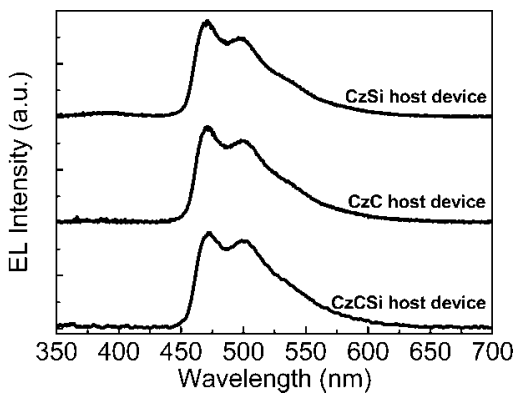

FIGURE 6. EL spectra of the devices.

(17), and LiF as the electron-injection layer (18). Chemical structures of related compounds and their energy levels are shown in Figure 5, in which ionization potentials (IPs) of host compounds $\mathrm{CzSi}, \mathrm{CzC}$, and $\mathrm{CzCSi}$ in films were determined by ultraviolet photoemission spectroscopy (UPS), while IPs of other materials were taken from our previous measurements or from the literature $(12,19)$. Electron affinities of all compounds were derived by subtracting IPs with optical energy gaps. As can be seen from the energy levels shown in Figure 5, either a trityl or triphenylsilyl substituent has a rather limited effect on the energy levels of the host compounds.

The EL spectra of the devices are shown in Figure 6, while Figure 7 depicts the $I-V-L$ characteristics and external EL quantum efficiencies of the devices. The devices employing the developed host materials all exhibit rather low turn-on voltages of $\sim 3 \mathrm{~V}$ (defined as the voltage where EL becomes detectable) and low operation voltages of $4.7-5.0 \mathrm{~V}$ at 100 $\mathrm{cd} / \mathrm{m}^{2}$, as shown in Figure $7 \mathrm{~b}$. High maximum external EL quantum efficiencies of $15.7 \%, 12.9 \%$, and $14.2 \%$ photons/ electron were obtained for the $\mathrm{CzSi}, \mathrm{CzC}$, and $\mathrm{CzCSi}$ devices, respectively. High quantum efficiencies along with the low operation voltages gave maximal power efficiencies of 26.7 , 25.9, and $25.0 \mathrm{~lm} / \mathrm{W}$ for the $\mathrm{CzSi}, \mathrm{CzC}$, and $\mathrm{CzCSi}$ devices, respectively. One important factor contributing to these improved characteristics is the judicious use of two holetransport layers (DPAS/TCTA) with a stepwise increase in the IPs to match those of the host materials and the choice of the electron-transport layer (TAZ). Use of only a single holetransport layer of either DPAS or TCTA results in lower efficiency and higher voltage. Adopting other electrontransport and hole-blocking layers widely used in phosphorescent OLEDs, such as 2,9-dimethyl-4,7-diphenyl-1,10- 

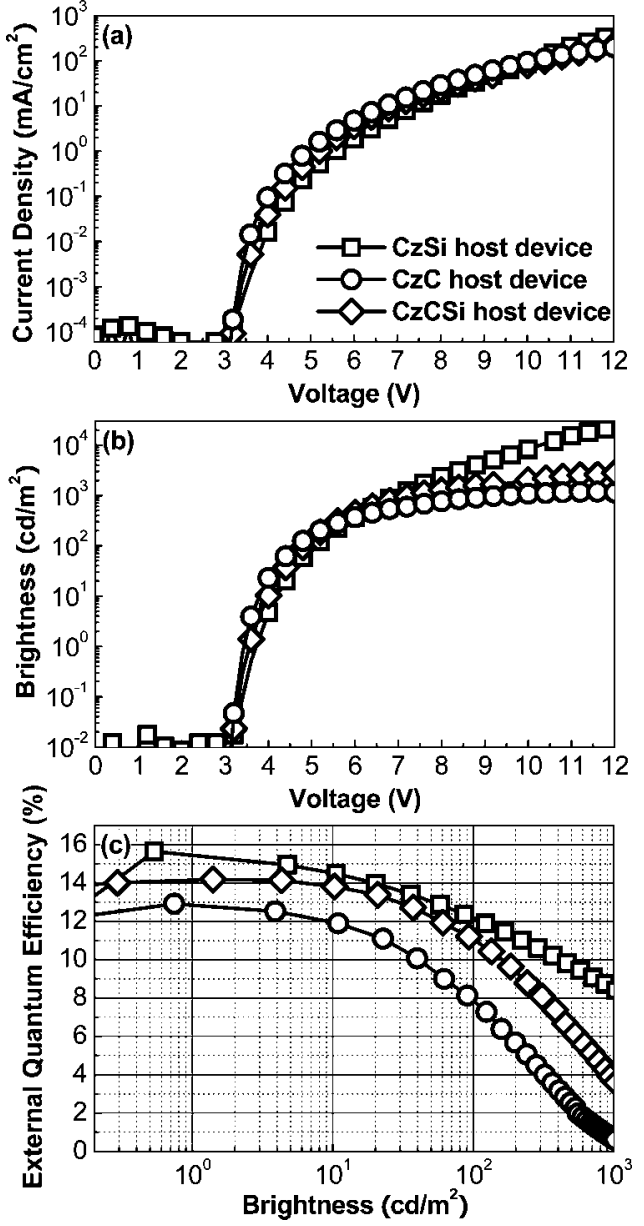

FIGURE 7. (a) $I-V$ characteristics, (b) $L-V$ characteristics, and (c) external EL quantum efficiency vs brightnesses of the devices.

phenanthroline, $2,2^{\prime}, 2^{\prime \prime}-(1,3,5$-benzenetriyl)tris [1-phenyl- $1 H$ benzimidazole], or 1,3-bis[2-(2,2'-bipyridin-6-yl)-1,3,4-oxadiazol-5-yl]benzene $(1-8,20)$, also substantially reduces the device efficiency. The rather small exchange energies of the developed carbazole-based host materials could be another factor that contributes to the improvement of the power efficiencies of the devices. It is also worth mentioning that although the phosphorescent dopant FIrpic emitting a less saturated blue light was used in this work, the developed host materials are expected to function as well for more saturated blue dopants considering their high triplet energies (21).

Although the performances of the devices using three different hosts, $\mathrm{CzSi}, \mathrm{CzC}$, and $\mathrm{CzCSi}$, are all similarly impressive in the lower-current-density region (i.e., the lower-brightness region), the device characteristics differ strongly at higher current densities. As can be seen from Figure $7 \mathrm{c}$, the $\mathrm{CzC}$ and $\mathrm{CzCSi}$ devices suffer from severe efficiency roll-off at higher current densities (i.e., at higher brightnesses) in comparison with the device using CzSi. While the CzSi device retains an efficiency of above $12 \%$ at $100 \mathrm{~cd} / \mathrm{m}^{2}$ and $9 \%$ at $1000 \mathrm{~cd} / \mathrm{m}^{2}$, the efficiency of the $\mathrm{CzC}$ device has dropped to $7 \%$ and $0.6 \%$ at 100 and $1000 \mathrm{~cd} /$ $\mathrm{m}^{2}$, respectively. Resistance against efficiency roll-off at high currents gives the $\mathrm{CzSi}$ device a maximum brightness as high as $59000 \mathrm{~cd} / \mathrm{m}^{2}$ at $14.5 \mathrm{~V}$. As for the $\mathrm{CzC}$ and $\mathrm{CzCSi}$ devices, the maximum brightnesses are only 2200 and $4200 \mathrm{~cd} / \mathrm{m}^{2}$, respectively. The superior performance of the CzSi host over the $\mathrm{CzC}$ and $\mathrm{CzCSi}$ analogues is most likely attributed to the differences in carrier-transport properties because other properties are similar among the three host materials. Efficiency roll-off at higher currents typically observed in phosphorescent OLEDs may be associated with triplet-triplet annihilation (1-8). With a relatively higher hole mobility in $\mathrm{CzC}$ and $\mathrm{CzCSi}$, the exciton formation regions of $\mathrm{CzC}$ and $\mathrm{CzCSi}$ are speculated to be highly concentrated at the vicinity of the interface between the emitting layer and electrontransport layer. Therefore, at high current densities and thus high exciton densities, the accumulated excitons suffer severe quenching by triplet-triplet annihilation, resulting in low efficiencies. On the contrary, the relatively lower hole mobility of $\mathrm{CzSi}$ (about 1 order lower than that of $\mathrm{CzC}$ ) could broaden the exciton distribution zone over the emitting layer, reducing the chance of quenching with high densities of triplet excitons. Such results demonstrate the significance of the thorough understanding and consideration of host material properties in the design of effective phosphorescent devices.

Such a difference in carrier-transport properties may also partly explain the difference between the devices using the $\mathrm{CzSi}$ host and the devices using the mCP host, in addition to their differences in morphological and electrochemical stabilities. In reported FIrpic/mCP devices with device structures similar to the one described in this work, they exhibited peak quantum efficiencies of $\sim 6-11 \%(6,7,22)$, significantly lower than the efficiency of the CzSi device. Meanwhile, $\mathrm{mCP}$ was reported to have a hole mobility of $5 \times 10^{-4}$ $\mathrm{cm}^{2} / \mathrm{V} \cdot \mathrm{s}(23)$, which is much higher than the hole mobility of $\mathrm{CzSi}\left(\sim 5 \times 10^{-5} \mathrm{~cm}^{2} / \mathrm{V} \cdot \mathrm{s}\right)$ and may also lead to highly concentrated carrier/concentrated distribution and degrade device performances (as in $\mathrm{CzC}$ and $\mathrm{CzCSi}$ ).

\section{CONCLUSIONS}

The development of effective host and dopant materials for blue phosphorescent OLEDs remains a challenge in OLED technology. Major issues for developing effective host materials used for blue electrophosphorescence are to satisfy many material requirements, such as large triplet energy, high thermal stability, electrochemical stability, tunable carrier-transport properties, etc. In this paper, we have performed systematic studies and demonstrated that these requirements can be achieved simultaneously by introducing nonconjugated, steric, bulky, and large-gap substitutions, such as triphenylsilyl and trityl groups, onto the electrochemically active $\mathrm{C} 3$ and $\mathrm{C} 6$ sites of carbazole. The developed compounds $\mathrm{CzSi}, \mathrm{CzC}$, and $\mathrm{CzCSi}$ preserve the large triplet energy of carbazole and yet exhibit much enhanced morphological and electrochemical stability. Most importantly, while these materials exhibit other similar physical and photophysical properties, such a molecular design strategy provides an extra capability of tuning carriertransport properties and provides a chance to study the significant influences of the carrier-transport properties on the device behaviors and performances. Such findings shall 
provide useful guidelines for the future design and selection of effective host materials for organic blue electrophosphorescence.

\section{EXPERIMENTAL SECTION}

Preparation of CzSi. 9-(4-tert-Butylphenyl)-3,6-dibromocarbazole $(4.57 \mathrm{~g}, 10 \mathrm{mmol})$ in THF $(250 \mathrm{~mL})$ was treated with $n$-BuLi (15.6 mL, 1.6 $\mathrm{M}$ in hexane, $25 \mathrm{mmol}$ ) at $-78{ }^{\circ} \mathrm{C}$ and quenched with a solution of chlorotriphenylsilane $(7.4 \mathrm{~g}, 25$ mmol) in THF (50 mL). The desired product was purified by column chromatography, eluting with $\mathrm{CHCl}_{3} /$ hexane $(1: 4)$ to provide the product as a white solid $(6.36 \mathrm{~g}, 78 \%): \mathrm{mp} 354{ }^{\circ} \mathrm{C}$ (DSC); IR (neat) $v 2960,2359,1681,1428,1260,1025,801$ 699, $512 \mathrm{~cm}^{-1} ;{ }^{1} \mathrm{H}$ NMR $\left(\mathrm{CDCl}_{3}, 400 \mathrm{MHz}\right) \delta 8.21(\mathrm{~s}, 2 \mathrm{H})$, $7.61-7.55(\mathrm{~m}, 16 \mathrm{H}), 7.49-7.41(\mathrm{~m}, 2 \mathrm{H}), 7.44-7.40(\mathrm{~m}, 8 \mathrm{H})$, $7.37-7.34(\mathrm{~m}, 12 \mathrm{H}) 1.42(\mathrm{~s}, 9 \mathrm{H}) ;{ }^{13} \mathrm{C} \mathrm{NMR}\left(\mathrm{CDCl}_{3}, 100 \mathrm{MHz}\right) \delta$ $150.1,141.5,136.0,134.4,134.0,133.4,129.0,128.5,127.4$ $126.3,126.1,123.5,122.7,109.5,35.1,31.7 ; \mathrm{MS}^{\left(\mathrm{m} / \mathrm{z}, \mathrm{FAB}^{+}\right)}$ 815(4). HRMS $\left(\mathrm{m} / \mathrm{z}, \mathrm{FAB}^{+}\right)$. Calcd for $\mathrm{C}_{58} \mathrm{H}_{49} \mathrm{NSi}_{2}: 815.3404$ Found: 815.3403. Elem anal. Calcd for $\mathrm{C}_{58} \mathrm{H}_{49} \mathrm{NSi}_{2}$ : C, 85.35; $\mathrm{H}$, 6.05. Found: C, 85.10; H, 6.07.

Preparation of CzC. 9-(4-tert-Butylphenyl)carbazole (2.99 g, $10 \mathrm{mmol}$ ) and triphenylmethanol $(5.47 \mathrm{~g}, 21 \mathrm{mmol})$ were dissolved in $\mathrm{CH}_{2} \mathrm{Cl}_{2}(70 \mathrm{~mL})$, and Eaton's reagent $(0.5 \mathrm{~mL}, 7.7$ wt \% $\mathrm{P}_{2} \mathrm{O}_{5}$ in $\mathrm{CH}_{3} \mathrm{SO}_{3} \mathrm{H}$ ) was added dropwise at $25^{\circ} \mathrm{C}$ under a nitrogen atmosphere. After stirring for $48 \mathrm{~h}$, the precipitate was filtered and washed with water $(50 \mathrm{~mL} \times 2)$ and a dilute aqueous sodium bicarbonate solution. The crude product suspended in acetone was refluxed for $1 \mathrm{~h}$ and refluxed for another $1 \mathrm{~h}$ in $\mathrm{CH}_{2} \mathrm{Cl}_{2}$. The white powder was filtered and dried: yield $6.6 \mathrm{~g}$ (84\%); $\mathrm{mp} 372-374^{\circ} \mathrm{C}$; IR (KBr) $v 3062,2963,1600,1520$, $1485,817,745,700 \mathrm{~cm}^{-1} ;{ }^{1} \mathrm{H}$ NMR $\left(\mathrm{CDCl}_{3}, 400 \mathrm{MHz}\right) \delta 7.79$ $(\mathrm{d}, J=1.6 \mathrm{~Hz}, 2 \mathrm{H}), 7.53(\mathrm{dd}, J=8.8$ and $2.0 \mathrm{~Hz}, 2 \mathrm{H}), 7.47(\mathrm{dd}$, $J=8.8 \mathrm{~Hz}, 2 \mathrm{H}), 7.30(\mathrm{~s}, 1 \mathrm{H}), 7.30-7.26(\mathrm{~m}, 4 \mathrm{H}), 7.26-7.16(\mathrm{~m}$, $27 \mathrm{H}), 7.12(\mathrm{dd}, J=8.8$ and $2.0 \mathrm{~Hz}, 2 \mathrm{H}), 1.40(\mathrm{~s}, 9 \mathrm{H}) ;{ }^{13} \mathrm{C} \mathrm{NMR}$ $\left(\mathrm{CDCl}_{3}, 100 \mathrm{MHz}\right) \delta 150.0,147.1,139.0,138.4,134.8,131.1$, $130.4,127.2,126.4,126.0,125.6,122.3,121.3,108.9,64.9$, 34.8, 31.5; MS (m/z, $\left.\mathrm{FAB}^{+}\right) 783(0.41)$. $\mathrm{HRMS}\left(\mathrm{M}^{+}, \mathrm{FAB}^{+}\right)$. Calcd for $\mathrm{C}_{60} \mathrm{H}_{49} \mathrm{~N}$ : 783.3865. Found: 783.3871. Elem anal. Calcd for $\mathrm{C}_{60} \mathrm{H}_{49} \mathrm{~N}$ : C, 91.91; H, 6.30; N, 1.79. Found: C, 91.97; H, 6.66; N, 1.48 .

Preparation of CzCSi. Under nitrogen, Eaton's reagent $(0.5$ $\mathrm{mL}$ ) was added into a solution of 9-(4-tert-butylphenyl)carbazole (2.99 g, $10 \mathrm{mmol})$ and triphenylmethanol (2.86 g, $11 \mathrm{mmol})$ dissolved in $\mathrm{CH}_{2} \mathrm{Cl}_{2}(70 \mathrm{~mL})$. After stirring for $48 \mathrm{~h}$, the crude product 3-trityl-9-(4-tert-butylphenyl)carbazole was subjected to silical gel column chromatography, eluting with $\mathrm{CHCl}_{3} /$ hexane (1:7) to provide the product 3-trityl-9-(4-tert-butylphenyl)carbazole (1; $3.52 \mathrm{~g}, 65 \%)$ as a white solid: $\mathrm{mp} 255-256^{\circ} \mathrm{C}$; IR (KBr) $v$ 3056, 2957, 1600, 1517, 1457, 1242, 742, $705 \mathrm{~cm}^{-1} ;{ }^{1} \mathrm{H} \mathrm{NMR}$ $\left(\mathrm{CDCl}_{3}, 400 \mathrm{MHz}\right) \delta 7.97(\mathrm{~d}, J=1.6 \mathrm{~Hz}, 1 \mathrm{H}), 7.92(\mathrm{~d}, J=8 \mathrm{~Hz}$, $1 \mathrm{H}), 7.54-7.52(\mathrm{~m}, 2 \mathrm{H}), 7.46-7.44(\mathrm{~d}, 2 \mathrm{H}), 7.40-7.27(\mathrm{~m}, 5 \mathrm{H})$, $7.25-7.21(\mathrm{~m}, 9 \mathrm{H}), 7.21-7.15(\mathrm{~m}, 6 \mathrm{H}), 1.40(\mathrm{~s}, 9 \mathrm{H}) ;{ }^{13} \mathrm{C} \mathrm{NMR}$ $\left(\mathrm{CDCl}_{3}, 100 \mathrm{MHz}\right) \delta 149.8,146.9,140.7,138.6,138.1,134.5$, $130.8,129.9,127.0,126.2,125.9,125.3,125.2,123.0,121.8$, $121.5,119.7,119.2,109.5,108.5,64.7,34.5,31.2 ; \mathrm{MS}(\mathrm{m} / \mathrm{z}$ $\mathrm{FAB}^{+}$) 541 (60). 3-Trityl-9-(4-tert-butylphenyl)carbazole (1.0 g, $1.8 \mathrm{mmol})$ was dissolved in $\mathrm{CH}_{2} \mathrm{Cl}_{2}(25 \mathrm{~mL})$. A solution of $\mathrm{N}$-bromosuccinimide $(0.33 \mathrm{~g}, 1.8 \mathrm{mmol})$ in dimethylformamide $(50 \mathrm{~mL})$ was added slowly at room temperature. After stirring for $4 \mathrm{~h}$, the precipitate was collected and washed with hexane $(50 \mathrm{~mL} \times 2)$ to provide the compound 3-bromo-6-trityl-9-(4-tertbutylphenyl)carbazole (2) as a white solid (1.0 g, 90\%): $\mathrm{mp}$ 317-319 ${ }^{\circ} \mathrm{C}$; IR (KBr) $v 3064,2967,1593,1518,1479,1247$, 816, 746, $705 \mathrm{~cm}^{-1}$; ${ }^{1} \mathrm{H}$ NMR $\left(\mathrm{CDCl}_{3}, 400 \mathrm{MHz}\right) \delta 8.02(\mathrm{~d}, J=2$ $\mathrm{Hz}, 1 \mathrm{H}), 7.93(\mathrm{~d}, J=1.6 \mathrm{~Hz}, 1 \mathrm{H}), 7.55-7.53(\mathrm{~m}, 2 \mathrm{H}), 7.42-7.38$ $(\mathrm{m}, 3 \mathrm{H}), 7.28-7.16(\mathrm{~m}, 18 \mathrm{H}) ;{ }^{13} \mathrm{C} \mathrm{NMR}\left(\mathrm{CDCl}_{3}, 100 \mathrm{MHz}\right) \delta$ 150.5, 147.0, 139.6, 139.2, 138.9, 134.3, 131.1, 128.1, 127.5,
127.4, 126.6, 126.1, 125.7, 125.1, 122.7, 121.7, 121.1, 112.3, $111.3,109.1,64.9,34.9,31.5 . n$-BuLi $(5.5 \mathrm{mmol}, 3.4 \mathrm{~mL}$ in a 1.6 M hexane solution) was added dropwise to a degassed THF $(50 \mathrm{~mL})$ solution of 3-bromo-6-(tripheylmethyl)-9-(4-tert-butylphenyl)carbazole $(3.1 \mathrm{~g}, 5 \mathrm{mmol})$ at $-78^{\circ} \mathrm{C}$. After stirring for $1 \mathrm{~h}$ at $-78^{\circ} \mathrm{C}$, chlorotriphenylsilane ( $\left.1.62 \mathrm{~g}, 5.5 \mathrm{mmol}\right)$ in THF $(30 \mathrm{~mL})$ was added in one portion. The resulting mixture was immediately warmed to room temperature. The precipitate was filtered and washed with hexane $(50 \mathrm{~mL})$ to provide a white powder of $\mathrm{CzCSi}(2.4 \mathrm{~g}, 60 \%)$ : $\mathrm{mp} 357-360{ }^{\circ} \mathrm{C}$; IR (KBr) $v 3071$, 2954, 1601, 1519, 1481, 1427, $1108,817,740,700 \mathrm{~cm}^{-1} ;{ }^{1} \mathrm{H}$ $\operatorname{NMR}\left(\mathrm{CDCl}_{3}, 400 \mathrm{MHz}\right) \delta 8.11(\mathrm{~s}, 1 \mathrm{H}), 7.88(\mathrm{~d}, J=1.2 \mathrm{~Hz}, 1 \mathrm{H})$, $7.61-7.59(\mathrm{~m}, 5 \mathrm{H}), 7.54(\mathrm{t}, 4 \mathrm{H}), 7.48(\mathrm{~s}, 2 \mathrm{H}), 7.46(\mathrm{~s}, 1 \mathrm{H}), 7.42$ $(\mathrm{dd}, J=7.8$ and $5.4 \mathrm{~Hz}, 5 \mathrm{H}), 7.36(\mathrm{t}, J=7 \mathrm{~Hz}, 7 \mathrm{H}), 7.3(\mathrm{~s}, 1 \mathrm{H})$, $7.28(\mathrm{~d}, J=5.6 \mathrm{~Hz}, 3 \mathrm{H}), 7.24-7.15(\mathrm{~m}, 10 \mathrm{H}), 1.40(\mathrm{~s}, 9 \mathrm{H}) ;{ }^{13} \mathrm{C}$ $\operatorname{NMR}\left(\mathrm{CDCl}_{3}, 100 \mathrm{MHz}\right) \delta 150.2,147.1,142.0,138.9,138.7$, 136.3, 134.8, 134.6, 133.4, 131.1, 130.6, 129.3, 128.6, 127.7, $127.2,126.5,126.1,125.7,123.3,123.2,122.0,121.6,109.7$, 108.9, 64.9, 34.8, 31.5; MS (m/z, $\left.\mathrm{FAB}^{+}\right) 799$ (100). Elem anal. Calcd: C, 88.57; H, 6.17; N, 1.75. Found: C, 88.58; H, 6.07; N, 1.38 .

Synthesized compounds were subjected to purification by temperature-gradient sublimation in a high vacuum before use in subsequent studies. CV was performed at a scan rate of 100 $\mathrm{mV} / \mathrm{s}$ using the glassy carbon electrode as the working electrode and $\mathrm{Ag} / \mathrm{AgCl}$ as the reference electrode. Oxidation $\mathrm{CV}$ was performed in $\mathrm{CH}_{2} \mathrm{Cl}_{2}$ with $0.1 \mathrm{M}$ of $n-\mathrm{Bu}_{4} \mathrm{NPF}_{6}$ as the supporting electrolyte.

Thermal properties of organic compounds were investigated by TGA and DSC. TGA was performed on a TA Instruments 2950 TGA thermal analyzer with a heating rate of $10^{\circ} \mathrm{C} / \mathrm{min}$ under nitrogen purging. DSC was performed on a TA Instruments DSC2920 low-temperature difference scanning calorimeter. During the DSC measurement, the sample was first heated at a rate of $20{ }^{\circ} \mathrm{C} / \mathrm{min}$ to melt, was quenched with liquid nitrogen, and then was heated again at $10^{\circ} \mathrm{C} / \mathrm{min}$ to record various transition temperatures.

Ultraviolet (UV) spectra were measured with a Shimadzu UV1601 PC spectrophotometer. Photolumenescence was measured using a CCD spectrograph and the $325 \mathrm{~nm}$ line of the $\mathrm{He}-\mathrm{Cd}$ laser as the excitation source. The phosphorescence spectra were obtained in dilute solutions at $77 \mathrm{~K}$. The emission spectra at $77 \mathrm{~K}$ were recorded with an IBH picosecond photon detection module and a sub-microsecond xenon flashlamp as an excitation source. Gated spectra were recorded to discriminate phosphorescence from fluorescence. The gate delay was $5 \mathrm{~ms}$ with a gate width of $5 \mathrm{~ms}$. The highest energy peak in the phosphorescence spectrum was taken for the $\mathrm{T}_{1} \rightarrow \mathrm{S}_{0}$ transition (i.e., the triplet energy).

Charge transport in films of various host materials was characterized by applying the TOF transient photocurrent technique on glass/Ag $(30 \mathrm{~nm}) /$ organic $(\sim 2 \mu \mathrm{m}) / \mathrm{Al}(150 \mathrm{~nm})$ samples in vacuum. Pulsed illumination (fourth harmonic of a Nd:YAG laser, $266 \mathrm{~nm}, 10 \mathrm{~ns})$ through the semitransparent electrode (Ag) induces photogeneration of a thin sheet of excess carriers. The sample thickness had been chosen to be much larger than the optical absorption depth of the excitation. Depending on the polarity of the applied bias, selected photogenerated carriers (holes or electrons) are swept across the sample with a transit time $t_{\mathrm{T}}$. With the applied bias $V$ and the sample thickness $D$, the applied electric field $E$ is then $V / D$, and the carrier mobility is given by $\mu=D / E t_{\mathrm{T}}=D^{2} / V t_{\mathrm{T}}$.

IPs of thin films of organic compounds were measured by UPS. The deposition and the UPS measurements of thin-film samples were performed in two interconnected ultrahighvacuum chambers. Organic thin films were deposited on goldcoated silicon substrates by thermal evaporation in the deposition chamber and then transferred in situ to the analysis chamber. In the analysis chamber with a base pressure of less 
than $1 \times 10^{-10}$ Torr, UPS was carried out using the He I ( 21.22 $\mathrm{eV})$ and He II (40.8 eV) photon lines and the double-pass cylindrical mirror analyzer to measure the energy spectrum of photoexcited electrons. The overall resolution of the UPS measurement is about $0.15 \mathrm{eV}$. The energy scale of UPS spectra is referenced to the Fermi level of the system, which is measured on the gold surface before deposition of organic thin films. IPs of molecular films can be deduced from the energy difference between the highest occupied molecular orbital level and the vacuum level (inferred from the low-energy onset of the UPS spectrum).

OLEDs were fabricated on the ITO-coated glass substrates with multiple organic layers sandwiched between the transparent bottom ITO anode and the top metal cathode. The material layers were deposited by vacuum evaporation in a vacuum chamber with a base pressure of $<10^{-6}$ Torr. The deposition system permits the fabrication of the complete device structure in a single vacuum pumpdown without breaking of the vacuum The deposition rate of organic layers was kept at $\sim 0.2 \mathrm{~nm} / \mathrm{s}$. The active area of the device is $1 \times 1 \mathrm{~mm}^{2}$, as defined by the shadow mask for cathode deposition. The $I-V-L$ ) characterization of the light-emitting devices was performed with a source measurement unit and a silicon photodiode calibrated with Photo Research PR650. EL spectra of devices were collected by a calibrated CCD spectrograph.

Acknowledgment. This study was supported financially by the National Science Council and Ministry of Economics of Taiwan.

\section{REFERENCES AND NOTES}

(1) Baldo, M. A.; O’Brien, D. F.; You, Y.; Shoustikov, A.; Thompson, M. E.; Forrest, S. R. Nature 1998, 395, 151.

(2) Adachi, C.; Baldo, M. A.; Thompson, M. E.; Forrest, S. R. J. Appl. Phys. 2001, 90, 5048.

(3) Adachi, C.; Kwong, R. C.; Djurovich, P.; Adamovich, V.; Baldo, M. A.; Thompson, M. E.; Forrest, S. R. Appl. Phys. Lett 2001, 79, 2082.

(4) Holmes, R. J.; Forrest, S. R.; Tung, Y.-J.; Kwong, R. C.; Brown, J. J.; Garon, S.; Thompson, M. E. Appl. Phys. Lett. 2003, 82, 2422.
(5) Tokito, S.; Iijima, T.; Suzuri, Y.; Kita, H.; Tsuzuki, T.; Sato, F. Appl. Phys. Lett. 2003, 83, 569

(6) Holmes, R. J.; D’Andrade, B. W.; Forrest, S. R.; Ren, X.; Li, J.; Thompson, M. E. Appl. Phys. Lett. 2003, 83, 3818.

(7) Yeh, S.-J.; Wu, M.-F.; Chen, C.-T.; Song, Y.-H.; Chi, Y.; Ho, M.-H.; Hsu, S.-F.; Chen, C.-H. Adv. Mater. 2005, 17, 285.

(8) Ren, X.; Li, J.; Holmes, R. J.; Djurovich, P. I.; Forrest, S. R.; Thompson, M. E. Chem. Mater. 2004, 16, 4743

(9) Avilov, I.; Marsal, P.; Brédas, J.-L.; Beljonne, D. Adv. Mater. 2004, 16,1624

(10) Brunner, K.; van Dijken, A.; Bömer, H.; Bastiaansen, J. J. A. M.; Kiggen, N. M. M.; Langeveld, B. M. W. J. Am. Chem. Soc. 2004, 126,6035

(11) Tsai, M.-H.; Hong, Y.-H.; Chang, C.-H.; Su, H.-C.; Wu, C.-C.; Matoliukstyte, A.; Simokaitiene, J.; Grigalevicius, S.; Grazulevicius, J. V.; Hsu, C.-P. Adv. Mater. 2007, 19, 862.

(12) Tsai, M.-H.; Lin, H.-W.; Su, H.-C.; Ke, T.-H.; Wu, C.-C.; Fang, F.C.; Liao, Y.-L.; Wong, K.-T.; Wu, C.-I. Adv. Mater. 2006, 18, 1216.

(13) Ambrose, J. F.; Carpenter, L. L.; Nelson, R. F. J. Electrochem. Soc. $1975,122,876$.

(14) Ambrose, J. F.; Nelson, R. F. J. Electrochem. Soc. 1968, 115, 1159.

(15) Frenkel, J. Phys. Rev. 1938, 54, 647.

(16) Elscher, A.; Bruder, F.; Heuer, H.-W.; Jonas, F.; Karbach, A.; Kirchmeyer, S.; Thurm, S.; Wehrmann, R. Synth. Met. 2000, 111, 139.

(17) Kido, J.; Kimura, M.; Nagai, K. Science 1995, 267, 1332.

(18) Hung, L. S.; Tang, C. W.; Mason, M. G. Appl. Phys. Lett. 1997, 70, 152.

(19) Adamovich, V. I.; Cordero, S. R.; Djurovich, P. I.; Tamayo, A.; Thompson, M. E.; D’Andrade, B. W.; Forrest, S. R. Org. Electron. 2003, 4, 77 .

(20) Ichikawa, M.; Kawaguchi, T.; Kobayashi, K.; Miki, T.; Furukawa, K.; Koyama, T.; Taniguchi, Y. J. Mater. Chem. 2006, 16, 221.

(21) Yang, C.-H.; Cheng, Y.-M.; Chi, Y.; Hsu, C.-J.; Fang, F.-C.; Wong, K.-T.; Chou, P.-T.; Chang, C.-H.; Tsai, M.-H.; Wu, C.-C. Angew. Chem., Int. Ed. 2007, 46, 2418.

(22) Holmes, R. J.; Forrest, S. R.; Tung, Y. J.; Kwong, R. C.; Brown, J. J.; Garon, S.; Thompson, M. E. Appl. Phys. Lett. 2003, 82, 2422.

(23) Wu, M.-F.; Yeh, S.-J.; Chen, C.-T.; Murayama, H.; Tsuboi, T.; Li, W.-S.; Chao, I.-T.; Liu, S.-W.; Wang, J.-K. Adv. Funct. Mater. 2007, $17,1887$.

AM800124Q 